\title{
Génétique et physiologie cellulaire
}

\section{Christine Petit}

\section{OpenEdition \\ Journals}

Édition électronique

URL : https://journals.openedition.org/annuaire-cdf/15551

DOI : 10.4000/annuaire-cdf.15551

ISBN : 978-2-7226-0572-5

ISSN : 2109-9227

Éditeur

Collège de France

Édition imprimée

Date de publication : 30 décembre 2020

Pagination : 121-123

ISBN : 978-2-7226-0516-9

ISSN : 0069-5580

Référence électronique

Christine Petit, "Génétique et physiologie cellulaire », L'annuaire du Collège de France [En ligne], 118 | 2020, mis en ligne le 01 avril 2021, consulté le 22 août 2022. URL : http://journals.openedition.org/ annuaire-cdf/15551 ; DOI : https://doi.org/10.4000/annuaire-cdf.15551 


\title{
GÉNÉTIQUE ET PHYSIOLOGIE CELLULAIRE
}

\author{
Christine PETIT \\ Membre de l'Institut (Académie des sciences), \\ professeure au Collège de France
}

Mots-clés : neurophysiologie auditive, surdités, thérapie

La série de cours et séminaires "Atteintes auditives : les thérapies du futur » est disponible, en audio et en vidéo, sur le site internet du Collège de France (https:// www.college-de-france.fr/site/christine-petit/course-2017-2018.htm).

\section{ENSEIGNEMENT}

COURS ET SÉMINAIRES - ATTEINTES AUDITIVES : LES THÉRAPIES DU FUTUR

\section{Cours 1 - Surdités : les leviers de l'innovation thérapeutique}

4 avril 2017

Séminaire 1 - Listening to a fast snail: Temporal processing in the auditory brainstem

Philipp Joris (professeur, Laboratory of Auditory Neurophysiology, Louvain, Belgique)

Cours 2 - Thérapie génique des surdités : (1) quid du potentiel des méthodes «traditionnelles »?

11 avril 2017

Séminaire 2 - From perception to pleasure: Music and its neural substrates

Robert Zatorre (professeur James McGill au Montreal Neurological Institute, McGill University/codirecteur de l'International Laboratory for Brain, Music, and Sound Research ou BRAMS) 
Cours 3 - Thérapie génique des surdités : (2) la chirurgie du génome, entre espoir et doute

6 juin 2017

\section{Séminaire 3 - From tempo to tears: Early level processing of sound rhythms in the mammalian brain}

Anna Montell Magnusson (professor, Audiology, Department of Clinical Science Intervention and Technology, Karolinska Institutet, Stockholm, Suède)

\section{Cours 4 - Thérapie cellulaire : transdifférenciation, cellules souches, la fabrique des organoïdes}

13 juin 2017

\section{Séminaire 4 - Towards the optical cochlear implant: Optogenetic stimulation of theauditory pathway}

Tobias Moser (professor, docteur, Institute for Auditory Neuroscience/InnerEarLab, University Medical Center, Göttingen, Allemagne)

\section{PuBLications}

2018

Bouzid A., Smeti I., Dhouib L., Roche M., Achour I., Khalfallah A., Gibriel A.A., Charfeddine I., Ayadi H., Lachuer J., Ghorbel A., Petit C. et Masmoudi S., « Downexpression of $P 2 R X 2, K C N Q 5, E R B B 3$ and SOCS3 through DNA hypermethylation in elderly women with presbycusis », Biomarkers, vol. 23, no 4, 2018, p. 347-356, DOI : 10.1080/1354750X.2018.1427795.

Dulon D., Papal S., Patni P., Cortese M., Vincent P.F.Y., Tertrais M., Emptoz A., Tlili A., Bouleau Y., Michel V., Delmaghani S., Aghaie A., Pepermans E., AlegriaPrevot O., Akil O., Lustig L., Avan P., Safieddine S., Petit C. et El-Amraoui A., "Clarin-1 gene transfer rescues auditory synaptopathy in model of Usher syndrome », The Journal of Clinical Investigation, vol. 128, no 8, 2018, p. 3382-3401, DOI : 10.1172/JCI94351.

PETIT C., « Génétique et physiologie cellulaire », Annuaire du Collège de France 2015-2016. Résumé des cours et travaux, vol. 116, 2018, p. 137-146, DOI : 10.4000/annuaire-cdf.12811; en ligne : https://journals.openedition.org/annuaire-cdf/12811.

Salime S., Riahi Z., Elrharchi S., Elkhattabi L., Charoute H., Nahili H., Rouba H., Kabine M., Bonnet C., PetiT C. et BaraKat A., «A novel mutation in SLITRK6 causes deafness and myopia in a Moroccan family », Gene, vol. 659, 2018, p. 89-92, DOI : 10.1016/j. gene.2018.03.042.

Talbi S., Bonnet C., Riahi Z., Boudjenah F., Dahmani M., Hardelin J.-P., Wong Jun TAi F., Louha M., Ammar-Khodja F. et PetiT C., «Genetic heterogeneity of congenital hearing impairment in Algerians from the Ghardaïa province », International Journal of Pediatric Otorhinolaryngology, vol. 112, 2018, p. 1-5, DOI : 10.1016/j.jporl.2018.06.012.

Trouillet A., Dubus E., Degardin J., Estivalet A., Ivkovic I., Godefroy D., GarcíaAyuso D., Simonutti M., Sahly I., Sahel J.A., El-Amraoui A., Petit C. et Picaud S., 
" Cone degeneration is triggered by the absence of USH1 proteins but prevented by antioxidant treatments », Scientific Reports, vol. 8, nº 1, 2018, p. 1968, DOI : 10.1038/s41598-018-20171-0.

\section{7}

Bahloul A., Pepermans E., Raynal B., Wolff N., Cordier F., England P., Nouaille S., Baron B., El-Amraoui A., Hardelin J.-P., Durand D. et Petit C., «Conformational switch of harmonin, a submembrane scaffold protein of the hair cell mechanoelectrical transduction machinery », FEBS Letters, vol. 591, no 15, 2017, p. 2299-2310, DOI : 10.1002/1873-3468.12729.

Delhommel F., Cordier F., Bardiaux B., Bouvier G., Colcombet-Cazenave B., Brier S., Raynal B., Nouaille S., Bahloul A., Chamot-Rooke J., Nilges M., Petit C. et WOLFF N., «Structural characterization of whirlin reveals an unexpected and dynamic supramodule conformation of its PDZ tandem », Structure, vol. 25, no 11, 2017, p. 1645-1656. e5, DOI : 10.1016/j.str.2017.08.013.

Emptoz A., Michel V., Lelli A., Akil O., Boutet de Monvel J., Lahlou G., Meyer A., Dupont T., Nouaille S., Ey E., Franca de Barros F., Beraneck M., Dulon D., Hardelin J.-P., Lustig L., Avan P., Petit C. et SAFIEDdine S., « Local gene therapy durably restores vestibular function in a mouse model of Usher syndrome type $1 \mathrm{G}$ », Proceedings of the National Academy of Sciences of the United States of America, vol. 114, $\mathrm{n}^{\circ}$ 36, 2017, p. 9695-9700, DOI : 10.1073/pnas.1708894114.

Michalski N., Goutman J.D., Auclair S.M., Monvel J.B. De, Tertrais M., EmPtoz A., Parrin A., Nouaille S., Guillon M., Sachse M., Ciric D., Bahloul A., Hardelin J.-P., Sutton R.B., Avan P., Krishnakumar S.S., Rothman J.E., Dulon D., SAFieddine S. et PETIT C., «Otoferlin acts as a $\mathrm{Ca}^{2+}$ sensor for vesicle fusion and vesicle pool replenishment at auditory hair cell ribbon synapses », ELife, vol. 6, 2017, p. e31013, DOI : 10.7554/eLife.31013. Michel V., Booth K.T., Patni P., Cortese M., Azaiez H., Bahloul A., Kahrizi K., labbe M., Emptoz A., Lelli A., Dégardin J., Dupont T., Aghaie A., OficjalskaPham D., Picaud S., Najmabadi H., Smith R.J., Bowl M.R., Brown S.D., Avan P., Petit C. et El-AmRaOuI A., «CIB2, defective in isolated deafness, is key for auditory hair cell mechanotransduction and survival », EMBO Molecular Medicine, vol. 9, $\mathrm{n}^{\circ}$ 12, 2017, p. 1711-1731, DOI : 10.15252/emmm.201708087.

Testa F., Melillo P., Bonnet C., Marcelli V., De Benedictis A., Colucci R., Gallo B., Kurtenbach A., Rossi S., Marciano E., Auricchio A., Petit C., Zrenner E. et SIMONELLI F., «Clinical presentation and disease course of usher syndrome because of mutations in MYO7A or USH2A », Retina, vol. 37, no 8, 2017, p. 1581-1590, DOI : 10.1097/ IAE.0000000000001389. 
